\title{
Factors and Interactions that Influence the Pressure Drop Across An Air Volume Reducing Device on Low-Pressure Water Distribution Networks
}

DOI: https://doi.org/10.1007/s40996-021-00682-z

\section{Authors}

Javier Carpintero, Fausto A. Canales, Jonathan Fábregas, José Ávila

\section{Abstract}

Efficient water supply systems are necessary for the development and sustainability of human societies. One relevant aspect of these systems is the metering function, recorded employing water meters, which determines the charges levied to the clients and estimates the water losses in the network. Inaccurate measurements are detrimental for both the client and the supplier. For allowing more precise metering, one option is to use an air volume reducing device, an accessory similar to a check valve that minimizes the air volume entrapped in the pipelines, thus improving metering accuracy. This research used an experimental design to determine the influence of four factors and their interactions on the pressure drop across these devices as a preliminary step for allowing their extended use on low-pressure water supply systems. The results showed that the diameter, the spring stiffness, and the flow rate are significant factors in the pressure drop. The shape of the valve stem is statistically significant only when interacting with other factors.

\section{Keywords}

Water metering, Design of experiments, Pressure drop, Valve diameter, Spring 